\title{
Emergence and Rearrangement of Dynamic Supramolecular Aggregates Visualised by Interferometric Scattering Microscopy
}

\author{
Maria A. Lebedeva, Elena Palmieri, Philipp Kukura, Stephen P. Fletcher*
}

Department of Chemistry, Chemistry Research Laboratory, University of Oxford, UK, OX1 3TA

*Correspondence to: stephen.fletcher@chem.ox.ac.uk

\begin{abstract}
Studying dynamic self-assembling systems in their native environment is essential for understanding the mechanisms of self-assembly and thereby exerting full control over these processes. Traditional ensemble-based analysis methods often struggle to reveal critical features of the self-assembly that occur at the single particle level. Here, we describe a label-free single-particle assay to visualise realtime self-assembly in aqueous solutions by interferometric scattering microscopy (iSCAT). We demonstrate how the assay can be applied to biphasic reactions yielding micellar or vesicular aggregates, detecting the onset of aggregate formation, quantifying the kinetics at the single particle level and distinguishing sigmoidal and exponential growth of aggregate populations. Furthermore, we can follow the evolution in aggregate size in real time, visualising the nucleation stages of the selfassembly processes and record phenomena such as incorporation of oily components into the micelle or vesicle lumen.
\end{abstract}

Key words: self-assembly; autocatalysis; single-particle imaging; interferometric scattering microscopy; label-free imaging

Self-assembly of soft materials in solution ${ }^{1}$ forms the basis of both physiological and pathological processes, ${ }^{2,3}$ nanoscience and manufacture of functional materials. ${ }^{4}$ The key step for assembly involves the aggregation, either in an ordered or in a disordered fashion, of solubilised molecules into nanoscopic structures, which can subsequently grow to the micro- or even mesoscale. These early aggregates, their constitution, shape and interactions critically influence the outcome of the assembly process, and thus constitute the basis of our mechanistic understanding of self-assembly. ${ }^{5-7}$ Our ability to directly visualise and monitor early-stage aggregates / assembly intermediates, however, has been limited due to their low abundance and small size, making their observation and quantification a significant experimental challenge. As a result, supramolecular assembly is traditionally studied using ensemble-based characterisation methods, which provide information about the average constitution and structure of aggregates. ${ }^{8}$ Such methods are very useful for identifying thermodynamic products, but have limited capacity for studying evolving or dynamic systems, and generally lack sensitivity during the critical nucleation stage of self-assembly. As a result, much effort has been aimed at the development of single particle analysis methods, to enable a more detailed mechanistic understanding of the underlying dynamics. The combination of automated experimentation, image processing, physicochemical analysis and machine learning allows significant advances to be made in understanding the driving forces of self-assembly in microscale systems. ${ }^{9,10}$ High speed atomic force microscopy (AFM) enables the visualisation of dynamic behaviour during molecular self-assembly, however it has limited temporal resolution and has to be performed on surfaces, so is unsuitable for solution measurements. ${ }^{11}$ Transmission electron microscopy (TEM), cryogenic (cryo-TEM) or Liquid 
cell (LCTEM) transmission electron microscopy can provide information about the structure and the morphology of the aggregates, ${ }^{12}$ but the preparation of samples involves drying, staining ${ }^{13}$ or cryogenic vitrification steps ${ }^{14}$ and the collection of images often leads to radiation damage to the sample. ${ }^{15,16}$ In addition, these techniques require careful data interpretation, as well as availability of complex instrumentation. ${ }^{17}$ Nanoparticle tracking analysis (NTA) enables study of aggregate size in solution at the single particle level. Detection of small particles smaller than about $40 \mathrm{~nm}$ is only possible for metallic nanoparticles. ${ }^{18,19}$ Super resolution fluorescence microscopy is a powerful method to analyse biological and synthetic ${ }^{20}$ self-assembly processes in solutions with excellent spatial and temporal resolution. ${ }^{21}$ However, it relies on the specimen being able to produce a fluorescence response. This can be achieved by exploiting aggregation induced emission (AIE), intrinsic to the selfassembling building blocks, ${ }^{22,23}$ or by incorporation of fluorescent labels, ${ }^{24}$ which is synthetically demanding, ${ }^{25}$ and may significantly interfere with the self-assembly process. There is therefore a clear need for methodologies that enable label-free, dynamic observation of single particle dynamics in real time in their native environment.

Interferometric scattering microscopy (iSCAT) has emerged as a powerful method for label-free imaging, detection and quantification of nanoscale objects in solutions, ${ }^{26-28}$ including the self-assembly of proteins into viral capsids. ${ }^{29}$ We have previously shown that it can detect micelles, and that it can be used to monitor the progress of a biphasic reaction producing aggregates in situ by performing the reaction directly on a microscope coverslip. ${ }^{30}$ However, the physical parameters of such in situ experiments are significantly different from those of traditional batch conditions. ${ }^{31} 1$. Only a few microliters of reactants/solutions are mixed in an in situ set up, whereas several millilitres are mixed in the reaction flask of a batch experiment. 2 . The surface to volume ratio, which is a crucial factor in biphasic reactions, is several orders of magnitude higher in in situ experiments than in batch experiments. 3. Stirring cannot be used in in situ experiments but is a constant parameter to control in batch reactions. 4. The temperature of the reaction mixture cannot be controlled in an in situ set up due to heating induced by the laser, and is presumably above room temperature, whereas it can be precisely controlled in batch experiments. All these factors make it impossible to correlate results obtained by iSCAT in the in situ set up with those from traditional batch experiments obtained by ensemble characterisation methods. In fact, the biphasic reactions studied in $\left[{ }^{30}\right]$ did not proceed under batch conditions, but only in situ on the iSCAT set-up, where the large surface-to-volume ratio presumably increased the contact area and accelerated the reaction between organic and aqueous components.

In contrast to our previous work, here we use iSCAT to study the evolution of aggregates under traditional batch conditions rather than in situ, and we present a quantitative comparison with traditional ensemble methods. Moreover, we demonstrate that in such systems iSCAT can not only detect and count particles, but also monitor their size evolution in time, which is crucial to understanding the mechanisms of self-assembly. Finally, we show that iSCAT can be used to study different types of supramolecular aggregates, not only micelles but also vesicles, which are more biologically relevant due to functions such as cellular transport and signalling. To achieve this, we studied two biphasic reactions involving formation of amphiphiles that self-assemble during the reaction and where there is feedback between the aggregates and the reaction rate. We characterized the kinetics of these reactions observing the emergence and evolution of aggregate particles in real time. The measurements revealed autocatalytic behaviour, formation and growth of micellar and vesicular aggregates, and supramolecular transformations occurring in these systems. 


\section{Results and discussion}

iSCAT visualises nanoscale objects in solution by monitoring particles that bind to a glass surface (Figure 1b). Binding aggregates change the local refractive index and therefore the scattering properties of the glass-water interface, which are quantified by iSCAT. The resulting images consist of diffraction-limited spots, with a ratiometric contrast value in the range of 0.001-0.1 (Figure 1). The number of particles binding to the glass surface over a given time interval is proportional to the particle concentration, whereas the particle contrast is proportional to the mass for a variety of biological particles, such as proteins, ${ }^{32}$ DNA, ${ }^{33}$ molecular machines, ${ }^{34}$ antibodies, ${ }^{35}$ protein cages ${ }^{36}$ and virus capsids ${ }^{29}$ (see Fig. S15 for contrast to mass calibration). At this stage, quantitative conversion of ratiometric contrast to particle size is not possible, however, previous studies of lipid nanodiscs showed that changes in lipid mass can be accurately quantified. ${ }^{32}$ Making the approximation that lipids produce a similar contrast to peptides based on these results, the correlation between ratiometric contrast of a supramolecular aggregate and its mass allows us to estimate the number of monomers that form the aggregate particle, and therefore quantify particle growth.

Our systems consist of a biphasic reaction between aqueous and organic components yielding amphiphiles capable of self-assembling into micelles or vesicles, performed under traditional batch conditions. These reactions occur at the interface of the aqueous and oily layer, where the hydrophilic and hydrophobic reactants come into contact. This reaction is initially slow, as it can only happen at the interface of the two phases. Therefore, any physical parameters that affect the mixing of the two phases (such as the size and shape of the reaction vessel, stirring speed and even the size, shape and orientation of the stirring bar) will affect the kinetics of surfactant formation. Achieving reproducibility thus requires precise control of these parameters. Once sufficient surfactant molecules are produced, they assemble into micelles or vesicles, which then accelerate the reaction by providing a larger interface surface area and by incorporating oily components, helping to mix the reactants. Although type and size of the aggregates that form in both in situ and bulk experiments are similar (see ESI page 4 and Fig. S3), the reaction kinetics differ. Therefore, we deliberately avoid studying the reaction in situ on the microscope to maintain reaction conditions identical to those used in bulk experiments and thus allow reliable comparison between bulk and single particle methods.

We first investigated a micelle-forming system (Figure 1a), where an amphiphilic product $\mathbf{3}$ forms through the biphasic reaction of aromatic disulphide 1, fully soluble in TRIS buffer, with 1-octanethiol 2. Compound $\mathbf{3}$ behaves as a physical autocatalyst ${ }^{37}$ by aggregating into small micelles at micromolar concentrations and enabling more efficient mixing of the phase-separated reagents. Destruction of metastable amphiphile $\mathbf{3}$ by an excess of thiol $\mathbf{2}$ would draw the system to its thermodynamic minimum, which can be avoided in out-of-equilibrium conditions. ${ }^{38}$ This work focuses on studying the system at early reaction stages, where the destruction step - slow compared to the formation of 3 is negligible.

Initially we analysed solutions of pure amphiphile $\mathbf{3}$ by iSCAT. 3 dissolves in $0.5 \mathrm{M}$ TRIS buffer at pH 7.5 yielding micellar aggregates, with a critical aggregation concentration (CAC) of $0.2 \mathrm{mM} .{ }^{38}$ Figure $1 \mathrm{c}$ shows the iSCAT contrast distribution of a $100 \mu \mathrm{M}$ solution of $\mathbf{3}$. The contrast distribution of micelles is slightly asymmetric, and to reflect this the data was fitted to a sum of two Gaussian functions, showing a peak contrast of the major component of at a contrast value of 0.006 , which corresponds 
to a protein mass of about $286 \mathrm{kDa}$ in the experimental setup used. Making the approximation that lipids produce a similar optical contrast to peptides in our setup, this would suggest about 800 molecules of 3 per micelle, given a molecular weight of $343 \mathrm{Da}$. Dynamic light scattering (DLS) of the same solution confirmed the presence of aggregates with hydrodynamic radii of approx. $5 \mathrm{~nm}$, which correlates well with the formation of small micelles (see SI Figure S2). It is worth noting that lipids containing a carboxylic acid group such as compound $\mathbf{3}$ may form either micelles or vesicles depending on the solution $\mathrm{pH} .{ }^{39,40}$ The exact $\mathrm{pH}$ range where one architecture dominates over the other depends on the electronic structure of the particular lipid. Compound $\mathbf{3}$ forms micelles of similar size at both pH 7.5 and pH 8 used this work (Fig. S2a and c in ESI). The chemistry of thiol-disulphide exchange is also affected by the $\mathrm{pH}$, with an acceleration at higher $\mathrm{pH}$ due to thiol deprotonation. Therefore, the choice of $\mathrm{pH}$ for each system reported here was dictated by the kinetics of the particular reaction.
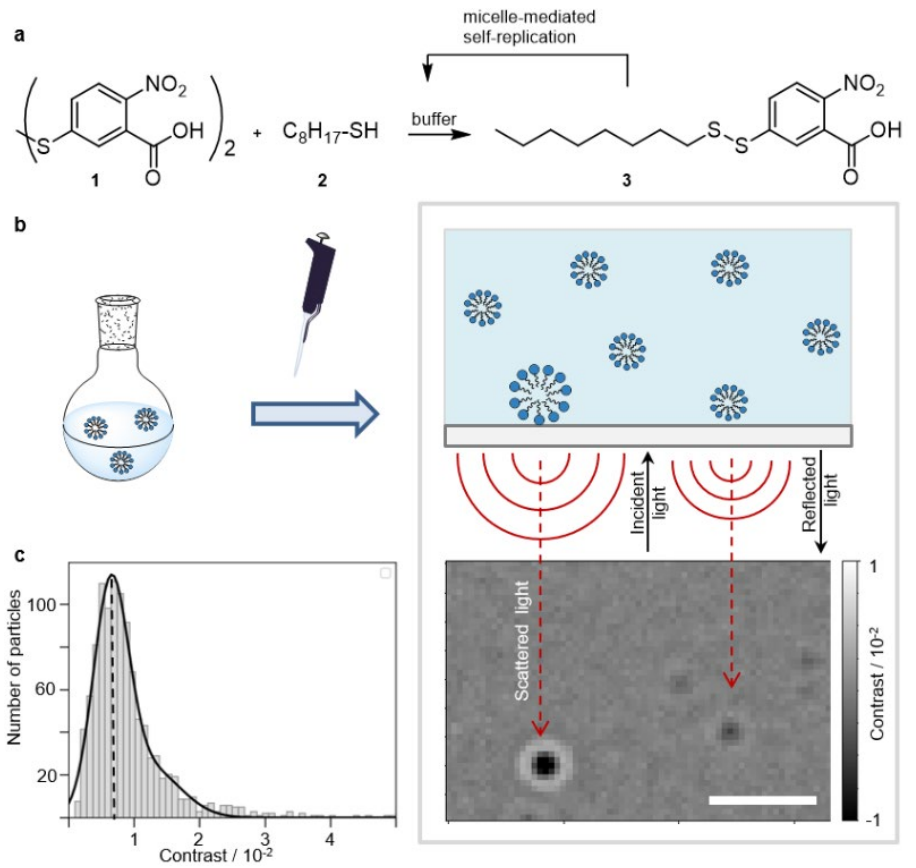

Figure 1. Principle of studying molecular self-assembly with interferometric scattering microscopy (iSCAT). a) A micelle-forming biphasic reaction between aromatic disulphide $\mathbf{1}$ and neat 1-octanethiol 2. b) Schematic of an iSCAT experiment showing the formation of micelles of $\mathbf{3}$ in a biphasic reaction under batch conditions, an aliquot of this solution being transferred to a microscope coverslip and directly measured by iSCAT; (right) individual micelles of $\mathbf{3}$ binding to the glass coverslip (top) and an iSCAT image produced by these micelles, with individual micelles visualised as dark circles (bottom). Scale bar: $1 \mu \mathrm{m}$. c) Ratiometric contrast histogram obtained from imaging a $100 \mu \mathrm{M}$ solution of $\mathbf{3}$ in $0.5 \mathrm{M}$ TRIS buffer at $\mathrm{pH}$ 7.5. The black line corresponds to a fit of the mass distribution to a sum of two Gaussians.

We then followed the formation of $\mathbf{3}$ in a biphasic reaction between $\mathbf{1}$ and $\mathbf{2}$ by taking $10 \mu \mathrm{L}$ aliquots from the aqueous layer at specific time intervals, and immediately recording $30 \mathrm{~s}$ videos on iSCAT (Figure $1 \mathrm{~b}$ ). The number of particles binding to the glass surface in each video was counted, and the contrast of each particle analysed using custom software written in Python. We varied the concentrations of $\mathbf{1}$ and $\mathbf{2}$, and we investigated the effect on reaction kinetics. In all experiments, the 
onset of particle formation is detected by the appearance of a small number of low contrast particles (Figure 2a and Supplementary video 1 ) followed by an increasing number of particles as the reaction progresses (Figure $2 \mathrm{~b}$ and $2 \mathrm{c}$ and Supplementary video 2 ). Figure $2 \mathrm{~d}$ shows the kinetic profile for formation of $\mathbf{3}$ under different reaction conditions, quantified by counting individual particles. The data can be fitted to a sigmoidal function, such as the Hill equation (see ESI page 6). We then used the geometrical approach to define the lag period as the intersection between initial and maximum slopes (or tangents to the curve). ${ }^{41,42}$ We observed a sigmoidal profile in all cases, with a lag period ranging between $4.5 \pm 0.5$ and $55 \pm 3 \mathrm{~min}$, depending on the concentration of 1 and the equivalents of 2 used (Figure 2e). This is consistent with autocatalytic behaviour previously reported for this system. ${ }^{38}$ Higher concentrations of 1 ( $2.5 \mathrm{mM}$ vs $1.25 \mathrm{mM}$ ) result in a shorter lag period (Figure $2 \mathrm{~d}$, blue vs orange line), as does excess of 2 (5 equiv. vs 1 equiv., Fig. $2 \mathrm{~d}$, brown vs orange line). The number of particles that can be detected by iSCAT is limited by the saturation of the glass coverslip surface with the aggregate particles. In order to avoid multiple binding events, i.e. more than one aggregate particle landing on the same diffraction-limited spot within the integration time $(0.1 \mathrm{~s})$, we monitored the reactions under comparatively dilute conditions. The possibility of each diffraction limited spot in the iSCAT video being attributed to more than one particle was excluded by considerations of landing rates similar to those described by Ortega-Arroyo et $a^{30}$ (see ESI page 6 for discussion). When the particle concentration exceeds this regime, dilution of aliquots can be used to further follow the reaction (see SI Figure S4a). Landing maps showing how densely the glass surface is covered by particles at different stages of the reaction are shown in Fig. S5. To compare our iSCAT data with an alternative ensemble measurement relying on scattering, we followed the evolution of aggregates by light scattering. We used a fluorescence spectrometer to monitor the scattering signal by setting the receiver wavelength identical to the incident light at $532 \mathrm{~nm}$, similar to the wavelength of the laser used in our iSCAT set-up $(520 \mathrm{~nm})$. In a reaction between $1.25 \mathrm{mM} 1$ and 1 equiv. 2 we observed a lag period of $15 \mathrm{~min}$ (see ESI Figure S6), comparable to that observed by iSCAT for the same reaction, thus confirming that our iSCAT method allows us to detect the onset of the lag period by single particle counting. 

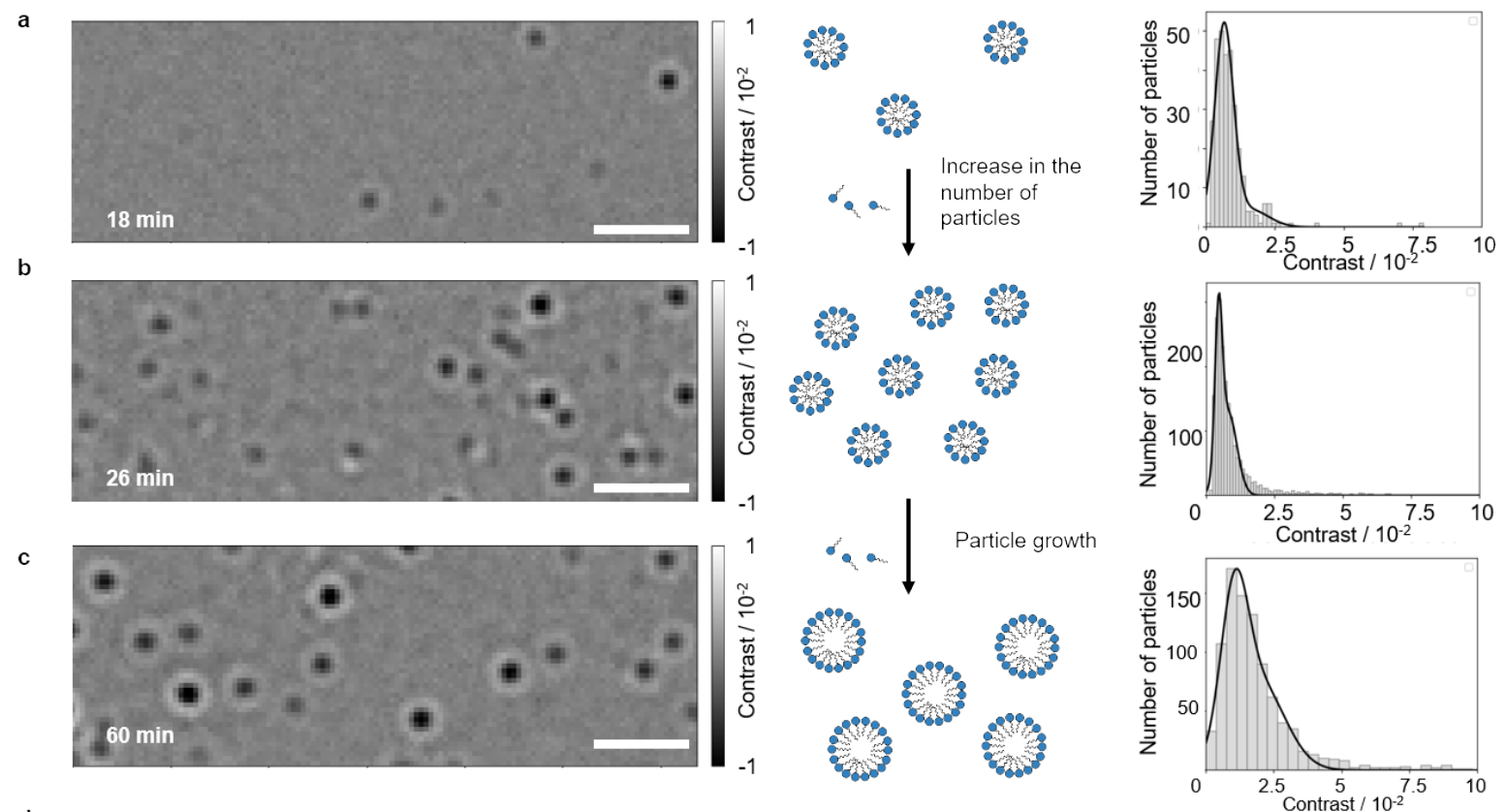

d

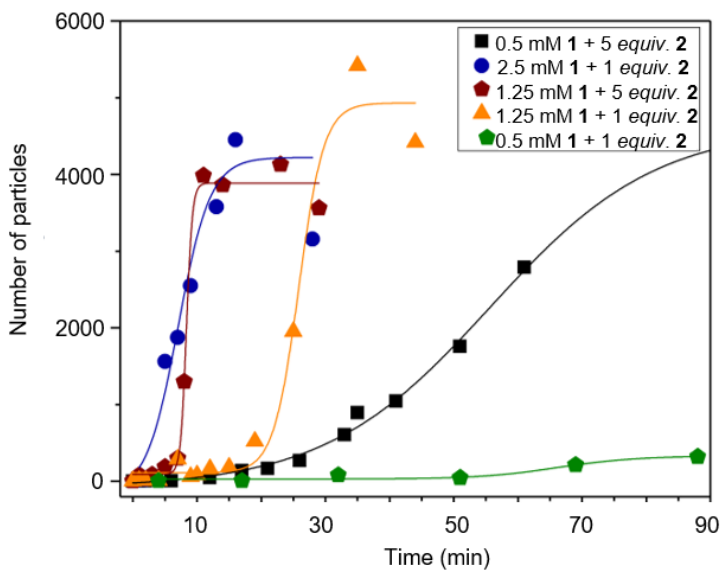

\begin{tabular}{|c|c|}
\hline Reaction & Lag period, $\min$ \\
\hline $0.5 \mathrm{mM} 1+1$ equiv. 2 & $55 \pm 3$ \\
\hline $0.5 \mathrm{mM} 1+5$ equiv. 2 & $37 \pm 7$ \\
\hline $1.25 \mathrm{mM} 1+1$ equiv. 2 & $20 \pm 2$ \\
\hline $1.25 \mathrm{mM} 1+5$ equiv. 2 & $6 \pm 1$ \\
\hline $2.5 \mathrm{mM} 1+1$ equiv. 2 & $4.5 \pm 0.5$ \\
\hline
\end{tabular}

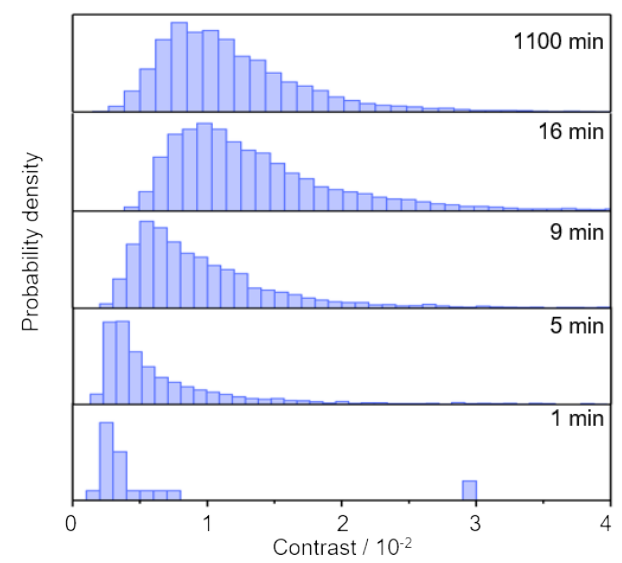

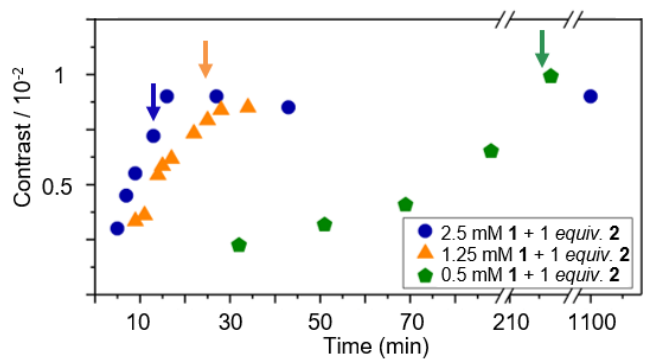

h

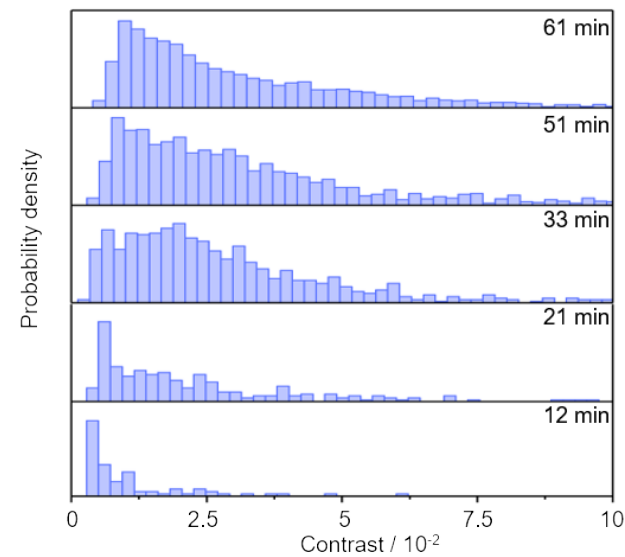

Figure 2. Monitoring micelle-forming biphasic reactions between $\mathbf{1}$ and $\mathbf{2}$ at the single particle level. a-c) iSCAT images of a reaction between $1.25 \mathrm{mM} 1$ and 1 equiv. 2 showing (a) low density of small particles at the onset of micelle formation, (b) increased density of small particles at a later stage and (c) high density of larger particles near completion, with the corresponding contrast distribution histograms (right). Scale bar: $1 \mu \mathrm{m}$; d) characterisation of the reaction kinetics by counting the number of landing events per $30 \mathrm{~s}$ per field of view in a biphasic reaction between $\mathbf{1}$ and $\mathbf{2}$ under different 
reaction conditions; data fitted to the Hill equation, showing a sigmoidal profile in each case; e) overview of the reaction kinetics obtained from these fits, each entry is an average of two experiments; f) change in the peak ratiometric contrast over time between $0.5 \mathrm{mM}, 1.25 \mathrm{mM}$ or 2.5 $\mathrm{mM} 1$ and 1 equiv. 2; arrows indicate the time points at which DLS analysis could be performed in each experiment; $g-h$ ) changes in the ratiometric contrast over the course of a biphasic reaction between (g) $2.5 \mathrm{mM} 1$ and 1 equiv. of $\mathbf{2}$ and (h) $0.5 \mathrm{mM} 1$ and 5 equiv. of 2 .

We can not only quantify the kinetics, but also monitor evolution of particle size with time. Figure $2 \mathrm{~g}$ shows the evolution of ratiometric contrast during the course of the reaction between $2.5 \mathrm{mM} 1$ and 1 equiv. of 2. Small aggregate particles begin to appear approximately $5 \mathrm{~min}$ after the reaction starts, and the system can be followed for another $15 \mathrm{~min}$ until the particle concentration becomes too high. Over this time period, the particle contrast increases from 0.003 ( $\sim 140 \mathrm{kDa}$ ) to 0.009 ( $\sim 30 \mathrm{kDa}$ ) (Fig. 2f). After $15 \mathrm{~min}$, dilution of an aliquot is required; $10 \mu \mathrm{L}$ aliquots are diluted with $90 \mu \mathrm{L}$ of $0.5 \mathrm{M}$ TRIS buffer and the resulting mixture is measured immediately to minimise equilibration and dissolution of micelles. These measurements showed no further increase of ratiometric contrast up to $t=1100 \mathrm{~min}$ (Figure $2 \mathrm{~g}$ and Fig. S7). Figure $2 \mathrm{f}$ summarises the change in the peak ratiometric contrast over time obtained from fitting to a sum of two Gaussians. In all experiments, the earliest detected particles are small ( 0.003 contrast; Fig. 2a and Supplementary video 1). This is approx. 3 times larger than the sensitivity limit of our set up (determined as $\sim 50 \mathrm{kDa}$, or $\sim 0.001$ contrast). A gradual growth of contrast is then observed until the thermodynamic micelles size, corresponding to $\sim 0.009$ contrast is reached, and then remains constant throughout the reaction (Fig. $2 \mathrm{c}$ and Supplementary video 2). This indicates that the assembly of surfactant $\mathbf{3}$ into micelles in the reaction proceeds via a nucleation step. The formation of seeds is visualised at the onset of the exponential growth stage in the kinetic profile. This nucleation process can be followed in time and depends on the concentration of 1 . For $2.5 \mathrm{mM} \mathbf{1}$ (Fig. $2 \mathrm{f}$, blue circles), the thermodynamic size is reached within $\sim 15 \mathrm{~min}$, and for $0.5 \mathrm{mM} 1$ (Fig. 2f, green pentagons) it is achieved in over $3 \mathrm{~h}$. This means that assembly of micelles proceeds gradually by formation of small aggregates at the beginning of the reaction, which then grow by incorporation of further surfactant until the most stable arrangement is achieved.

We compared our results to those obtained by following the reaction under the same conditions by DLS. As DLS is a bulk analysis technique and iSCAT is a single particle method, the two techniques are intrinsically different and provide somewhat different information. DLS quantifies the average scattering properties of the aggregate solution, and therefore the average size of the particles, whereas iSCAT operates at the single particle level. In our systems, detecting particles and their size at the nucleation stages of the reaction by DLS was not possible due to insufficient DLS sensitivity. Signal sufficient for DLS analysis only appears at the later stages, marked by arrows on Fig. $2 \mathrm{f}$, when an iSCAT contrast of 0.009 (and thus thermodynamic size of the aggregates) and an increase in particle concentration is reached. For example, in a reaction between $1.25 \mathrm{mM} 1$ and 1 equiv. 2, a signal sufficient for DLS analysis appears only after $30 \mathrm{~min}$ and shows the presence of 5-7 nm micelles (Fig. S9a), similar to that of $\mathbf{3}$ in pure buffer (Fig. S2). This means that the initial nucleation step corresponding to $<0.009$ contrast cannot be observed by DLS. This shows that iSCAT detects particles at much lower concentrations than DLS. This is advantageous for studying very dilute systems, or very early stages of self-assembly where aggregates emerge gradually over time. Furthermore, DLS is a lowresolution method ${ }^{43}$ in the absence of additional separation steps. Another advantage of iSCAT is the time scale of the experiments. We can measure changes in the reaction every $30 \mathrm{~s}$, therefore resolving changes in the aggregate number and size on a sub-minute time scale. DLS data is usually acquired 
over longer time periods. For instance, the lag period in some of our reactions is $4.5 \mathrm{~min}$, which can be measured by iSCAT but is difficult to resolve by DLS. Finally, DLS measurements are very sensitive to temperature and solvent viscosity. Therefore, temperature must be kept constant and solvent viscosity must be known for reliable DLS experiments, ${ }^{43}$ whereas iSCAT does not require controlling temperature or any prior knowledge of solvent/buffer viscosity.

We next investigated the size evolution of particles when an excess of thiol is used. Under these conditions, slow destruction of $\mathbf{3}$ is expected, however we focus our investigations on the early stages of the reaction, when destruction of $\mathbf{3}$ is still negligible. In a reaction between $0.5 \mathrm{mM} 1$ and 5 equiv. of $\mathbf{2}$ (Figure $2 \mathrm{~h}$ ), small micellar aggregates with contrast $\sim 0.003$ were observed in the first $20 \mathrm{~min}$. The contrast distribution then becomes more heterogeneous, tailing far into the high contrast region up to 0.1 contrast, over 10 times larger than the average contrast for pure micelles (supplementary video 3). We can see the gradual emergence of large aggregates over time, as well as the presence of small micelles with a contrast below 0.01 at all times. DLS measurements of the reaction mixture performed after 60 min also show the presence of larger aggregates with hydrodynamic radii of 35 nm (Figure S9b). These large aggregates most likely form by incorporation of the excess thiol present under reaction conditions. These observations were independent of the concentrations of $1(0.5 \mathrm{mM}$ and $1.25 \mathrm{mM}$ ) (Fig. S8 c-d). We performed control experiments where excess 2 (5 equiv.) was added to a $0.5 \mathrm{mM}$ solution of $\mathbf{3}$, and the mixture was stirred at $100 \mathrm{rpm}$, replicating the reaction conditions. A significant increase of ratiometric contrast was observed over $30 \mathrm{~min}$ (see SI, Figure S10), showing a broader contrast distribution, and contrast values tailing up to 0.1 , confirming that the increase in micelle size is likely due to incorporation of thiol $\mathbf{2}$. Under these conditions, slow destruction of $\mathbf{3}$ by the excess of $\mathbf{2}$ is expected, and iSCAT data after $18 \mathrm{~h}$ do not show any aggregate particles in either reaction mixture or control experiment.

Micelles play an important role in stabilising oil-water interfaces in both synthetic and biological processes. Vesicles are also important, due to their biological functions, such as cellular transport and signalling. To test the applicability of iSCAT to study both micelles and vesicles, we applied it to a similar vesicle-forming autocatalytic system. Reaction between an aromatic disulphide $\mathbf{4}$ and thiol $\mathbf{2}$ yields amphiphile 5 (Figure 3a), which in $0.25 \mathrm{M}$ HEPES buffer at $\mathrm{pH} 8$ forms aggregates with hydrodynamic radii of $40 \mathrm{~nm}$, as measured by DLS (Fig. S2b). These aggregates are most likely vesicles due to their large size and formation of an opaque solution, characteristic of vesicles. Ratiometric contrast histogram of $100 \mu \mathrm{M} 5$ in $0.25 \mathrm{M} \mathrm{HEPES}$ buffer at $\mathrm{pH} 8$ exhibit a contrast of $\sim 0.04$, significantly larger than micelles (this corresponds to a $1.7 \mathrm{MDa}$ protein, or, based on a $490 \mathrm{Da}$ monomer mass of $\mathbf{5}$, to ca. 3500 molecules of $\mathbf{5}$ in a vesicle) (Figure 3c). We compared these results with a typical vesicleforming lipid, DOPC, which showed similar contrast distribution histogram (see SI Figure S14). 


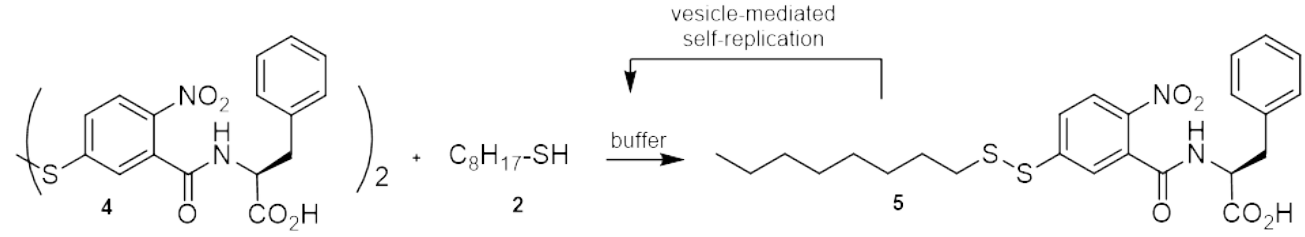

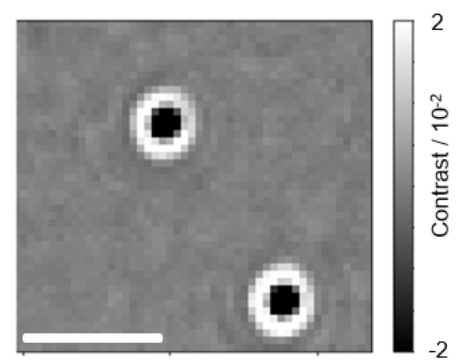

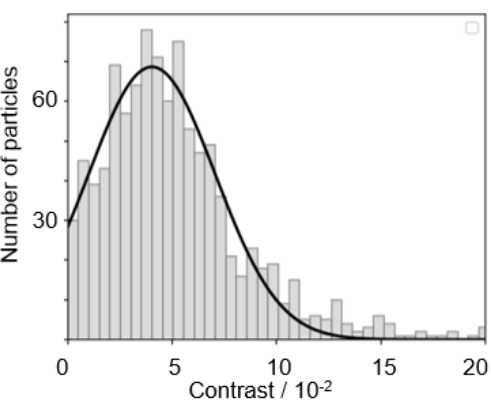

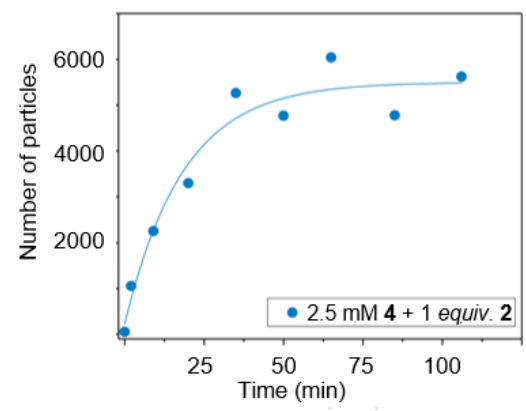

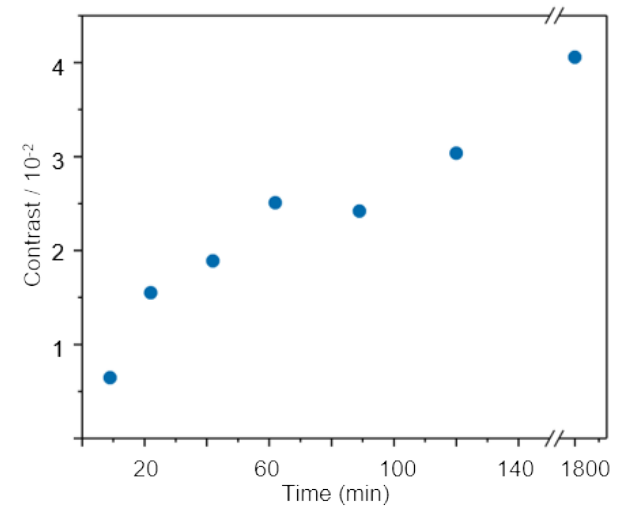

f

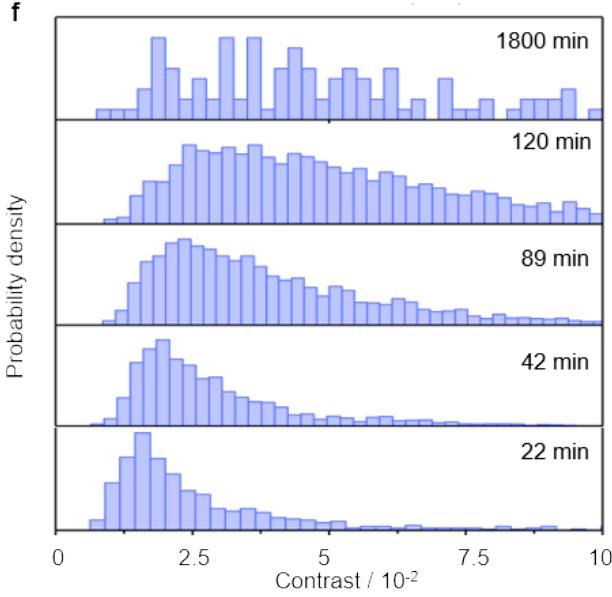

Figure 3. Monitoring vesicle-forming biphasic reactions between $\mathbf{4}$ and $\mathbf{2}$ by iSCAT. a) Schematic of a vesicle-forming biphasic reaction between aromatic disulphide 4 and neat 1-octanethiol 2. b) iSCAT image of the vesicles of 5. Scale bar: $1 \mu \mathrm{m}$. c) Ratiometric contrast histogram obtained from imaging a $100 \mu \mathrm{M}$ solution of 5 in 0.25 M HEPES buffer at pH 8 fitted to a Gaussian function. d) Characterisation of reaction kinetics by counting the number of landing events per $30 \mathrm{~s}$ per field of view showing an exponential increase in particle number. e-f) Changes in the ratiometric contrast over the course of a biphasic reaction between $2.5 \mathrm{mM} 4$ and 1 equiv. of 2 showing (e) the increase of the peak contrast over time and (f) overall changes in the contrast distribution.

The assay was performed as described above. Aggregate formation was observed almost immediately, and we could not record a lag period using iSCAT (Figure 3d). This is consistent with our kinetic studies on this system by traditional ensemble methods, where we could observe a very short lag period only by fine-tuning the experimental conditions (see ESI, Figure S13 and the relevant discussion). The iSCAT kinetics show a sharp increase in the number of particles, which can be fitted to an exponential growth function (Figure 3d). This behaviour differs from the sigmoidal growth observed in a reaction between 1 and 2 initially producing micelles. We ascribe this to differences in the critical aggregation concentration (CAC) ( $200 \mu \mathrm{M}$ for 3 and $\sim 5-10 \mu \mathrm{M}$ for 5, see SI Figure S11). Low CAC of 5 allows nearly instantaneous formation of aggregates, which accelerate the reaction thus showing exponential growth.

The size distribution of aggregates of $\mathbf{5}$ was also different. In the early stages of the reaction, we observed small aggregates with contrast values of $<0.01$, comparable to those previously measured 
for micelles (Fig. 3e-f). However, we found a gradual evolution of particle size reaching contrast values of 0.03-0.05 after 2 hours - nearly 10 times higher than those observed for micelles, and consistent with formation of vesicles (Supplementary video 4). The shape of the contrast distribution histogram features a gradually elongating tail in the high contrast region implying polydispersity in particle sizes, which is often observed in vesicles. Our studies suggest that, also in this system, early stages of selfassembly involve a nucleation stage with formation of small aggregates, which then grow into larger vesicles. The exact mechanisms of vesicles growth have been a subject of debate for years. ${ }^{39,40}$ The growth of unilamellar vesicles may proceed by incorporating more lipid molecules into the existing bilayer, thus forming bigger unilamellar vesicles. Alternatively, vesicles may grow by forming an entirely new bilayer around the existing one, resulting in multilamellar vesicles. At this stage, we are not able to distinguish the two plausible mechanisms by iSCAT. Detailed investigations on how reactant concentration affects the vesicles growth could shed light onto the mechanisms of vesicle formation and growth, and will form the basis of future work.

\section{Conclusions}

In this work, we have developed an assay for monitoring the formation and evolution of supramolecular aggregates in their native environment using iSCAT microscopy. The assay investigates self-assembly at the single particle level thus providing mechanistic details of self-assembly which are often concealed by ensemble analysis methods. The assay is label-free and therefore can be performed on any supramolecular aggregates without the need for synthetic modification of the building blocks. The assay reveals valuable information about the supramolecular assembly of amphiphiles into micelles and vesicles during the course of biphasic reactions. We can precisely measure the onset of micelle and vesicle formation and quantify the reaction kinetics, distinguishing between sigmoidal and exponential growth in autocatalytic reactions.

Moreover, we can follow the evolution of aggregate size in time and visualise the intermediates emerging at the early stages of the assembly process. We have detected that both micelles and vesicle formation proceeds through a nucleation step in which the particle size is significantly smaller than the average aggregate size at later stages. Both micelles and vesicles then show gradual concentrationdependent growth of aggregates after the initial nucleation events until a thermodynamic size of the aggregate is achieved. Furthermore, control experiments allowed us to detect incorporation of oily reaction components into micelles, which has been long postulated as a mechanism for autocatalysis. ${ }^{44}$

This label-free approach to studying complex dynamic self-assembly processes in solution is of particular interest, because it can directly visualise and quantify the molecular self-assembly intermediates at various stages of the reaction at the single particle level. Currently the sensitivity of iSCAT allows to detect and quantitatively measure the mass of particles with an optical contrast equivalent to a $50 \mathrm{kDa}$ protein or larger, regardless of the particle shape. iSCAT analysis requires minimal amounts of sample, with the working range from $\sim 30 \mathrm{pM}$ to $\sim 100 \mathrm{nM}$ particle concentrations, and $\sim 10 \mu \mathrm{L}$ of solution per measurement. It enables kinetic probing for self-assembly processes with a time-resolution on the order of 30 seconds, in the range of minutes to hours.

In future work, we aim to use the assay to follow the assembly of aggregates in complex multicomponent reactions. We anticipate that this will enable us to determine whether different types of aggregates can emerge simultaneously from complex mixtures of precursors, and real time examination of correlations between product distribution and aggregate size. The assay will disclose information otherwise inaccessible on phenomena such as selection and reproduction of aggregates 
in competitive environment, uptake and release of molecular components in the micelle or vesicle lumen, as well as vesicles growth, division and self-reproduction relevant to the emergence of life.

\section{Methods}

General details. Reagents $\mathbf{1}$ and $\mathbf{2}$ and TRIS and HEPES buffers were obtained from Sigma Aldrich and used without further purification. Preparation of buffers and synthesis of compounds 3-5 is described in SI.

Biphasic reactions between 1 and 2. A $5 \mathrm{mM}$ stock solution of 1 ( $25 \mathrm{~mL}$ ) in TRIS buffer was prepared by dissolving 1 ( $42.8 \mathrm{mg}, 0.12 \mathrm{mmol}$ ) in $0.5 \mathrm{M}$ TRIS buffer $\mathrm{pH}$ 8. The solution was briefly sonicated to break any remaining undissolved particles, and the $\mathrm{pH}$ was adjusted to 7.5 with $12 \mathrm{M} \mathrm{HCl}$. The solution was filtered through a $0.2 \mu \mathrm{m}$ cellulose filter and stored at $4^{\circ} \mathrm{C}$.

In a typical biphasic reaction, an aliquot of stock solution was placed in a $10 \mathrm{~mL}$ round bottom flask (RBF) and diluted with TRIS buffer pH 7.5 to $2.5 \mathrm{mM}, 1.25 \mathrm{mM}$ or $0.5 \mathrm{mM}$ concentrations, to make 5 $\mathrm{mL}$ solutions of 1. 1-Octanethiol 2 (1 equiv. or 5 equiv.) was then carefully added to the top of the aqueous solution. The reaction mixture was stirred at $100 \mathrm{rpm}$, and $10 \mu \mathrm{L}$ aliquots were taken from the aqueous layer at given time intervals for iSCAT analysis.

Biphasic reactions between 4 and 2 were set similarly. A $5 \mathrm{mM}$ stock solution of 4 ( $25 \mathrm{~mL}$ ) in HEPES buffer was prepared by dissolving $4(61.2 \mathrm{mg}, 0.12 \mathrm{mmol})$ in $0.25 \mathrm{M}$ HEPES buffer $\mathrm{pH}$ 8. The solution was briefly sonicated and filtered through a $0.2 \mu \mathrm{m}$ cellulose filter. An aliquot of stock solution was placed in a $10 \mathrm{~mL}$ RBF and diluted with HEPES buffer $\mathrm{pH} 8$ to a $2.5 \mathrm{mM}$ concentration, to make $2 \mathrm{~mL}$ of solution of 4. 1-Octanethiol 2 (1 equiv.) was then carefully added to the top of the aqueous solution. The reaction mixture was stirred at $100 \mathrm{rpm}$, and $10 \mu \mathrm{L}$ aliquots were taken from the aqueous layer at given time intervals for iSCAT analysis.

Interferometric scattering microscopy (iSCAT). The iSCAT experimental set-up is nearly identical to that described by Young et al, ${ }^{32}$ using a $520 \mathrm{~nm}$ diode laser used as the incident light source. Frames were recorded at $1 \mathrm{kHz}$ with an exposure time of $0.98 \mathrm{~ms}$, using a CMOS camera. Focus in the $z$ axis was maintained using an autofocus system relying on the total internal reflection (TIRF) of a $638 \mathrm{~nm}$ beam. Instrument control was performed using the custom software written in LabView.

Data processing. Data processing was performed using custom software written in Python, as described elsewhere. ${ }^{32}$ In brief, differential imaging was achieved by subtracting sets of images temporally offset by a time $\Delta$ t. The signal-to-noise ratio was then improved by spatially ( $3 \times 3$ binning) and temporally averaging the differential images (50 images). Particle detection was performed as described by Young et al. ${ }^{32}$ Briefly, diffraction-limited spots were identified by the software, and fitted to the 2D Gaussian function to give the ratiometric contrast value. The possibility of each diffraction limited spot being attributed to more than one particle was excluded by considerations of landing rates similar to those described by Ortega-Arroyo et al. ${ }^{30}$

Coverslips and sample preparation for iSCAT analysis. Samples for iSCAT analysis were prepared using ultrapure MilliQ water and filtered through $0.2 \mu \mathrm{m}$ cellulose filters. Reaction mixture aliquots were analysed as obtained, without further purification. 
Glass coverslips (no. 1.5, $24 \times 50 \mathrm{~mm}$, VWR; and $24 \times 24 \mathrm{~mm}$, VWR) were cleaned by sequential sonication in MilliQ water, isopropanol and MilliQ water ( 5 min each), and dried under the stream of nitrogen. Clean coverslips were assembled into flow chambers using double-sided-sticky tape (3M) as described by Young et al. ${ }^{32}$ Fresh aluminium foil was folded around an A4 size cutting board. Individual $24 \times 24$ coverslips were taped using two strips of double-sided tape and cut from the foil using a scalpel blade. Each excised $24 \times 24$ coverslip was joined, tape side down, in the center of a $24 \times 50$ coverslip and stored prior to use.

\section{Acknowledgements}

S.P.F. thanks the EPSRC (EP/M0025241/1) and ERC (Consolidator Grant, Autocat, 681491) for funding. P.K. is supported by an ERC (Consolidator Grant, Photomass, 819593).

Supporting Information Available online: supplementary videos 1-4 showing micelles of $\mathbf{3}$ and vesicles of $\mathbf{5}$ evolved under different conditions; compounds synthesis and characterisation; ${ }^{1} \mathrm{H},{ }^{13} \mathrm{C}$ NMR and HRMS spectra; dynamic light scattering and fluorimetric scattering; additional data on quantification of kinetics and evolution of particle size by iSCAT; characterisation of a biphasic reaction between 4 and 2 by ensemble methods; contrast to mass calibration.

\section{References.}

1. Whitesides, G. M.; Grzybowski, B. Self-Assembly at All Scales. Science 2002, 295, 2418-2421.

2. Philp, D.; Fraser Stoddart, J. Self-Assembly in Natural and Unnatural Systems. Angew. Chem. Int. Ed. 1996, 35, 1154-1196.

3. Petkau-Milroy, K.; Brunsveld, L. Supramolecular Chemical Biology; Bioactive Synthetic SelfAssemblies. Org. Biomol. Chem. 2013, 11, 219-232.

4. Amabilino, D. B.; Smith, D. K.; Steed, J. W. Supramolecular Materials. Chem. Soc. Rev. 2017, 46, 2404-2420.

5. Biswal, D.; Kusalik, P. G. Probing Molecular Mechanisms of Self-Assembly in Metal-Organic Frameworks. ACS Nano 2017, 11, 258-268.

6. Chowdhury, A. U.; Taylor, G. J.; Bocharova, V.; Sacci, R. L.; Luo, Y.; McClintic, W. T.; Ma, Y. Z.; Sarles, S. A.; Hong, K.; Collier, C. P.; Doughty, B. Insight into the Mechanisms Driving the SelfAssembly of Functional Interfaces: Moving from Lipids to Charged Amphiphilic Oligomers. J. Am. Chem. Soc. 2020, 142, 290-299.

7. Ogi, S.; Stepanenko, V.; Sugiyasu, K.; Takeuchi, M.; Würthner, F. Mechanism of Self-Assembly Process and Seeded Supramolecular Polymerization of Perylene Bisimide Organogelator. J. Am. Chem. Soc. 2015, 137, 3300-3307.

8. Patterson, J. P.; Robin, M. P.; Chassenieux, C.; Colombani, O.; O’Reilly, R. K. The Analysis of Solution Self-Assembled Polymeric Nanomaterials. Chem. Soc. Rev. 2014, 43, 2412-2425.

9. Points, L. J.; Taylor, J. W.; Grizou, J.; Donkers, K.; Cronin, L. Artificial Intelligence Exploration of Unstable Protocells Leads to Predictable Properties and Discovery of Collective Behavior. Proc. Natl. Acad. Sci. U. S. A. 2018, 115, 885-890.

10. Taylor, J. W.; Eghtesadi, S. A.; Points, L. J.; Liu, T.; Cronin, L. Autonomous Model Protocell Division Driven by Molecular Replication. Nat. Commun. 2017, 8, 4-9.

11. Fukui, T.; Uchihashi, T.; Sasaki, N.; Watanabe, H.; Takeuchi, M.; Sugiyasu, K. Direct Observation and Manipulation of Supramolecular Polymerization by High-Speed Atomic Force Microscopy. Angew. Chem. Int. Ed. 2018, 57, 15465-15470. 
12. Wang, H.; Li, B.; Kim, Y. J.; Kwon, O. H.; Granick, S. Intermediate States of Molecular SelfAssembly from Liquid-Cell Electron Microscopy. Proc. Natl. Acad. Sci. U. S. A. 2020, 117, 12831292.

13. Friedrich, H.; Frederik, P. M.; De With, G.; Sommerdijk, N. A. J. M. Imaging of Self-Assembled Structures: Interpretation of TEM and Cryo-TEM Images. Angew. Chem. Int. Ed. 2010, 49, 7850-7858.

14. Löbling, T. I.; Haataja, J. S.; Synatschke, C. V.; Schacher, F. H.; Müller, M.; Hanisch, A.; Gröschel, A. H.; Müller, A. H. E. Hidden Structural Features of Multicompartment Micelles Revealed by Cryogenic Transmission Electron Tomography. ACS Nano 2014, 8, 11330-11340.

15. Touve, M. A.; Carlini, A. S.; Gianneschi, N. C. Self-Assembling Peptides Imaged by Correlated Liquid Cell Transmission Electron Microscopy and MALDI-Imaging Mass Spectrometry. Nat. Commun. 2019, 10, 4837.

16. Ross, F. M. Opportunities and Challenges in Liquid Cell Electron Microscopy. Science 2015, 350, aaa9886-1-9.

17. De Yoreo, J. J.; N. A. J. M., S. Investigating Materials Formation with Liquid-Phase and Cryogenic TEM. Nat. Rev. Mater. 2016, 1, 16035.

18. Shang, J.; Gao, X. Nanoparticle Counting: Towards Accurate Determination of the Molar Concentration. Chem. Soc. Rev. 2014, 43, 7267-7278.

19. Kim, A.; Ng, W. B.; Bernt, W.; Cho, N. J. Validation of Size Estimation of Nanoparticle Tracking Analysis on Polydisperse Macromolecule Assembly. Sci. Rep. 2019, 9, 1-14.

20. Aliprandi, A.; Mauro, M.; De Cola, L. Controlling and Imaging Biomimetic Self-Assembly. Nat. Chem. 2016, 8, 10-15.

21. Huang, B.; Bates, M.; Zhuang, X. Super-Resolution Fluorescence Microscopy. Annu. Rev. Biochem. 2009, 78, 993-1016.

22. Peng, H. Q.; Liu, B.; Wei, P.; Zhang, P.; Zhang, H.; Zhang, J.; Li, K.; Li, Y.; Cheng, Y.; Lam, J. W. Y.; Zhang, W.; Lee, C. S.; Tang, B. Z. Visualizing the Initial Step of Self-Assembly and the Phase Transition by Stereogenic Amphiphiles with Aggregation-Induced Emission. ACS Nano 2019, 13, 839-846.

23. Li, J.; Li, Y.; Chan, C. Y. K.; Kwok, R. T. K.; Li, H.; Zrazhevskiy, P.; Gao, X.; Zhi Sun, J.; Qin, A.; Tang, B. Z. An Aggregation-Induced-Emission Platform for Direct Visualization of Interfacial Dynamic Self-Assembly. Angew. Chem. Int. Ed. 2014, 53, 13518-13522.

24. Albertazzi, L.; van der Zwaag, D.; Leenders, C. M. A.; Fitzner, R.; van der Hofstad, R. W.; Meijer, E. W. Probing Exchange Pathways in One-Dimensional Aggregates with Super-Resolution Microscopy. Science 2014, 344, 491-495.

25. Toseland, C. P. Fluorescent Labeling and Modification of Proteins. J. Chem. Bio. 2013, 6, 8595.

26. Amos, L. A.; Amos, W. B. The Bending of Sliding Microtubules Imaged by Confocal Light Microscopy and Negative Stain Electron Microscopy. J. Cell Sci. 1991, 98, 95-101.

27. Lindfors, K.; Kalkbrenner, T.; Stoller, P.; Sandoghdar, V. Detection and Spectroscopy of Gold Nanoparticles Using Supercontinuum White Light Confocal Microscopy. Phys. Rev. Lett. 2004, 93, 037401-1.

28. Kukura, P.; Ewers, H.; Müller, C.; Renn, A.; Helenius, A.; Sandoghdar, V. High-Speed Nanoscopic Tracking of the Position and Orientation of a Single Virus. Nat. Methods 2009, 6, 923-927. 
29. Garmann, R. F.; Goldfain, A. M.; Manoharan, V. N. Measurements of the Self-Assembly Kinetics of Individual Viral Capsids around Their RNA Genome. Proc. Natl. Acad. Sci. U. S. A. 2019, 116, 22485-22490.

30. Ortega-Arroyo, J.; Bissette, A. J.; Kukura, P.; Fletcher, S. P. Visualization of the Spontaneous Emergence of a Complex, Dynamic, and Autocatalytic System. Proc. Natl. Acad. Sci. U. S. A. 2016, 113, 11122-11126

31. Bissette, A. J.; Odell, B.; Fletcher, S. P. Physical Autocatalysis Driven by a Bond-Forming ThiolEne Reaction. Nat. Commun. 2014, 5, 4607.

32. Young, G.; Hundt, N.; Cole, D.; Fineberg, A.; Andrecka, J.; Tyler, A.; Olerinyova, A.; Ansari, A.; Marklund, E. G.; Collier, M. P.; Chandler, S. A.; Tkachenko, O.; Allen, J.; Crispin, M.; Billington, N.; Takagi, Y.; Sellers, J. R.; Eichmann, C.; Selenko, P.; Frey, L. et al. Quantitative Mass Imaging of Single Biological Macromolecules. Science 2018, 360, 423 LP - 427.

33. Li, Y.; Struwe, W. B.; Kukura, P. Single Molecule Mass Photometry of Nucleic Acids. Nucleic Acids Research 2020, gkaa632.

34. Sonn-Segev, A.; Belacic, K.; Bodrug, T.; Young, G.; VanderLinden, R. T.; Schulman, B. A.; Schimpf, J.; Friedrich, T.; Dip, P. V.; Schwartz, T. U.; Bauer, B.; Peters, J. M.; Struwe, W. B.; Benesch, J. L. P.; Brown, N. G.; Haselbach, D.; Kukura, P. Quantifying the Heterogeneity of Macromolecular Machines by Mass Photometry. Nat. Commun. 2020, 11, 1-26.

35. Soltermann, F.; Foley, E. D. B.; Pagnoni, V.; Galpin, M.; Benesch, J. L. P.; Kukura, P.; Struwe, W. B. Quantifying Protein-Protein Interactions by Molecular Counting with Mass Photometry. Angew. Chem. Int. Ed. 2020, 59, 10774-10779.

36. Malay, A. D.; Miyazaki, N.; Biela, A.; Chakraborti, S.; Majsterkiewicz, K.; Stupka, I.; Kaplan, C. S.; Kowalczyk, A.; Piette, B. M. A. G.; Hochberg, G. K. A.; Wu, D.; Wrobel, T. P.; Fineberg, A.; Kushwah, M. S.; Kelemen, M.; Vavpetič, P.; Pelicon, P.; Kukura, P.; Benesch, J. L. P.; Iwasaki, K. et al. An Ultra-Stable Gold-Coordinated Protein Cage Displaying Reversible Assembly. Nature 2019, 569, 439-442.

37. Bissette, A. J.; Fletcher, S. P. Mechanisms of Autocatalysis. Angew. Chem. Int. Ed. 2013, 52, 12800-12826.

38. Morrow, S. M.; Colomer, I.; Fletcher, S. P. A Chemically Fuelled Self-Replicator. Nat. Commun. 2019, 10, 1011.

39. Stano, P.; Wehrli, E.; Luisi, P. L. Insights into the Self-Reproduction of Oleate Vesicles. J. Phys. Condens. Matter 2006, 18, S2231-S2238.

40. Zhu, T. F.; Szostak, J. W. Coupled Growth and Division of Model Protocell Membranes. J. Am. Chem. Soc. 2009, 131, 5705-5713.

41. Baty, F.; Delignette-Muller, M. L. Estimating the Bacterial Lag Time: Which Model, Which Precision? Int. J. Food Microbiol. 2004, 91, 261-277.

42. Bentea, L.; Watzky, M. A.; Finke, R. G. Sigmoidal Nucleation and Growth Curves Across Nature Fit by the Finke-Watzky Model of Slow Continuous Nucleation and Autocatalytic Growth: Explicit Formulas for the Lag and Growth Times Plus Other Key Insights. J. Phys. Chem. C 2017, 121, 5302-5312.

43. Stetefeld, J.; McKenna, S. A.; Patel, T. R. Dynamic Light Scattering: A Practical Guide and Applications in Biomedical Sciences. Biophys. Rev. 2016, 8, 409-427.

44. Buhse, T.; Nagarajan, R.; Lavabre, D.; Micheau, J. C. Phase-Transfer Model for the Dynamics of “Micellar Autocatalysis." J. Phys. Chem. A 1997, 101, 3910-3917. 